\title{
Identificação do diagnóstico de enfermagem autocontrole ineficaz da saúde em alcoolistas: um estudo descritivo
}

\author{
Identifying the nursing diagnosis ineffective self-health management among alcoholics: a descriptive study
}

Identificación del diagnóstico de enfermería Gestión ineficaz de la propia salud en alcohólicos: un estudio descriptivo

Alexciane Priscila da Silva ${ }^{1}$, Jaqueline Galdino Albuquerque Perrelli², Fernanda Jorge Guimarães ${ }^{3}$, Suzana de Oliveira Mangueira ${ }^{4}$, Simara Lopes Cruz ${ }^{5}$, Iracema da Silva Frazão ${ }^{6}$

\footnotetext{
${ }^{1}$ Enfermeira. Vitória de Santo Antão, PE, Brasil. E-mail: alexcia silva@hotmail.com.

${ }^{2}$ Enfermeira, Mestre em Enfermagem. Discente do Programa de Pós-Graduação em Neuropsiquiatria e Ciências do Comportamento, nível Doutorado, da Universidade Federal de Pernambuco (UFPE). Professora Assistente da UFPE. Vitória de Santo Antão, PE, Brasil. E-mail: jaquealbuquerque@terra.com.br.

${ }^{3}$ Enfermeira, Mestre em Enfermagem. Discente do Programa de Pós-Graduação em Enfermagem, nível Doutorado, da Universidade Federal do Ceará (UFC). Professora Assistente da UFPE. Vitória de Santo Antão, PE, Brasil. E-mail: ferjorgui@hotmail.com.

${ }^{4}$ Enfermeira, Mestre em Enfermagem. Discente do Programa de Pós-Graduação em Enfermagem, nível Doutorado, da UFC. Professora Assistente da UFPE. Vitória de Santo Antão, PE, Brasil. E-mail: suzanaom@hotmail.com.

${ }^{5}$ Fonoaudióloga, Mestre em Saúde Pública. Professora Assistente da UFPE. Vitória de Santo Antão, PE, Brasil. E-mail: simara.cruz@hotmail.com.

${ }^{6}$ Enfermeira, Doutora em Serviço Social. Professora Adjunta da UFPE. Recife, PE, Brasil. E-mail: isfrazao@gmail.com.
}

\section{RESUMO}

O Alcoolismo é um problema de saúde pública que exige importante atuação do enfermeiro na prestação de cuidados sistematizados. Objetivou-se identificar o diagnóstico de enfermagem Autocontrole Ineficaz da Saúde em alcoolistas. Trata-se de um estudo descritivo, tipo transversal, desenvolvido em um hospital localizado no interior do estado de Pernambuco. A amostra foi de 46 pacientes. A coleta dos dados foi realizada por meio de entrevista estruturada com a utilização de um instrumento com informações sobre o diagnóstico de enfermagem em estudo. Identificou-se o Autocontrole Ineficaz da Saúde em 28,3\% dos pacientes alcoolistas. Esse fenômeno apresentou relação com as características Escolhas na vida diária ineficazes para atingir as metas de saúde, Expressão de desejo de controlar a doença e Falha em agir para reduzir fatores de risco. Os achados sugerem a necessidade da reestruturação do cuidado de enfermagem de acordo com essas características.

Descritores: Alcoolismo; Processos de Enfermagem; Diagnóstico de Enfermagem; Cooperação do Paciente.

\section{ABSTRACT}

Alcoholism is a public health issue requiring important intervention from nurses providing systemized care. The objective was to identify the nursing diagnosis ineffective self-health management among alcoholics. This descriptive, cross-sectional study was performed at a hospital located in Pernambuco state. The sample consisted of 46 patients. Data collection was performed by means of structured interviews using an instrument containing information about the referred nursing diagnosis. The diagnosis Ineffective Self-Health management was identified in $28.3 \%$ of the alcoholic patients. A relationship was observed between this phenomenon and the following characteristics: Daily life choices ineffective to achieve health goals, Expression of a wish to manage the disease and Failure in reducing risk factors. The findings suggest there is a need to restructure nursing care according o these characteristics.

Descriptors: Alcoholism; Nursing Process; Nursing Diagnosis; Patient Compliance.

\section{RESUMEN}

El alcoholismo es un problema de salud pública que exige importante actuación del enfermero en la prestación de cuidados sistematizados. Se objetivó identificar el diagnóstico de enfermería Gestión ineficaz de la propia salud en alcohólicos. Estudio descriptivo, de tipo transversal, desarrollado en hospital ubicado en el interior del estado de Pernambuco. La muestra fue de 46 pacientes. Los datos fueron recolectados mediante entrevista estructurada, utilizando instrumento con informaciones sobre el diagnóstico de enfermería en estudio. Se identificó Gestión ineficaz de la propia salud en $28,3 \%$ de los pacientes alcohólicos. Tal fenómeno presentó relación con las características En su vida diaria hace elecciones ineficaces para alcanzar objetivos de salud, Verbaliza deseos de manejar la enfermedad y Fracaso al emprender acciones para reducir los factores de riesgo. Los hallazgos sugieren la necesidad de reestructurar el cuidado de enfermería de acuerdo a tales características.

Descriptores: Alcoholismo; Procesos de Enfermería; Diagnóstico de Enfermería; Cooperación del Paciente. 


\section{INTRODUÇÃO}

O uso abusivo de álcool e outras drogas é um fenômeno de distribuição mundial e suas consequências são de relevância social. O Alcoolismo está entre as maiores causas de mortes em todo mundo, superado apenas pelas doenças cardíacas e pelo câncer. Estima-se que o uso prejudicial do álcool seja responsável por 2,5 milhões de óbitos a cada ano. Assim, 4\% de todas as mortes são atribuíveis ao uso dessa substância. Isso o torna um grave problema de saúde pública(1).

Dados do II Levantamento Nacional sobre os padrões do consumo dessa substância na população brasileira, realizado em 2012 , mostraram que $48 \%$ das pessoas com idade acima de 18 anos já consumiram bebida alcoólica, pelo menos uma vez ao ano. Ademais, $16 \%$ faz uso nocivo e $17 \%$ da população de bebedores apresentou critérios para abuso e/ou dependência de álcool(2).

Quanto aos efeitos prejudiciais, 32\% dos adultos que bebem referiram dificuldades para cessar o consumo, $10 \%$ relataram episódios com violência, $8 \%$ admitiram efeito prejudicial no trabalho e $9 \%$ afirmaram contexto familiar prejudicado devido ao consumo de álcool(2).

Segundo a Classificação Internacional de Doenças, em sua versão 10 (CID-10), a Síndrome da Dependência Alcoólica é definida como: "Conjunto de fenômenos comportamentais, cognitivos e fisiológicos que se desenvolvem depois de repetido consumo de uma substância psicoativa"(3).

Diante da concepção da multifatoriedade que envolve o Alcoolismo e de acordo com o preconizado pela Política Nacional sobre Drogas, a atenção ao usuário de álcool deve ser abrangente, com inserção do sujeito dependente nas esferas de prevenção, tratamento, recuperação, reinserção social e ocupacional, considerando a redução de danos como lógica norteadora ${ }^{(4)}$.

Do ponto de vista assistencial, o tratamento ao usuário de substâncias psicoativas (SPA) tem passado por mudanças significativas em seu modelo de atenção à saúde, antes centrado nos hospitais psiquiátricos. Nos últimos 11 anos, o Brasil tem implementado uma série de políticas de atendimento em saúde mental e na área de Álcool e Outras Drogas que trouxeram uma nova perspectiva em termos de atenção integral ao usuário de SPA $^{(4)}$.
A portaria $n^{\circ} 3088$ de 2011, republicada em maio de 2013, instituiu a Rede de Atenção Psicossocial com a finalidade de criar, ampliar e articular os pontos de atenção à saúde para pessoas com sofrimento ou transtorno mental e com necessidades decorrentes do uso de crack, álcool e outras drogas, no âmbito do Sistema Único de Saúde (SUS). A rede é composta por serviços de atenção primária à saúde, Centros de Atenção Psicossocial (CAPS) nas suas diferentes modalidades, serviços de urgência e emergência, atenção residencial de caráter transitório, atenção hospitalar, estratégias de desinstitucionalização e reabilitação psicossocial(5).

Contudo, mesmo diante da implementação destas políticas públicas, a literatura aponta que o alcoolista e sua família enfrentam uma série de obstáculos referentes à busca por tratamento, adesão e manutenção. As recaídas, o desejo de beber, a dificuldade de reconhecer o seu padrão de consumo como problemático e danoso a sua saúde e de toda a família são alguns fatores importantes que interferem no sucesso do tratamento(6).

Um estudo realizado com base em prontuários com 227 usuários de um CAPS -Álcool e outras Drogas (AD) mostrou que $50,6 \%$ não procuraram tratamento por iniciativa própria ${ }^{(7)}$. Esse fato mostra a importância de se conhecer quais os fatores que interferem nesse processo de tomada de decisão, bem como na adesão e no progresso do tratamento.

Quanto ao cuidado de enfermagem ao paciente alcoolista, o enfermeiro fundamenta a sua prática por meio do Processo de Enfermagem. Trata-se de um método que estimula o pensamento crítico, o julgamento clínico sobre todas as informações acerca da situação de saúde dos sujeitos e coletividades e orienta o processo de tomada de decisão de forma consciente para alcançar excelentes resultados que qualifiquem e descrevam as ações desse profissional nas mais variadas situações de prática(8).

Neste estudo, optou-se por estudar a ocorrência do Diagnóstico de Enfermagem Autocontrole Ineficaz da Saúde com o intuito de verificar quais os principais aspectos que influenciam a adesão do paciente alcoolista ao tratamento. Esse fenômeno é definido pela Classificação da NANDA-I (North American Nursing Association International), versão 2012-2014, como "padrão de regulação e integração à vida diária de um regime terapêutico para tratamento de doenças e suas 
sequelas que é insatisfatório para alcançar as metas específicas de saúde"(9). Salienta-se que esse diagnóstico, antes denominado Controle Ineficaz do Regime Terapêutico, sofreu modificações em seu enunciado, a partir da versão 2009-2011, porém suas características definidoras e fatores relacionados permaneceram inalterados.

Pesquisas sobre a identificação desse fenômeno em amostras de pessoas diabéticas ${ }^{(10-11)}$ mostraram que déficit de conhecimento, complexidade do sistema de assistência à saúde e déficit de apoio social são aspectos importantes a serem considerados quando se trata da capacidade do indivíduo de cuidar da sua saúde. Não foram encontradas investigações desse diagnóstico em amostras significativas de pacientes alcoolistas.

Diante da complexidade da problemática do alcoolismo torna-se fundamental a identificação dos fatores que influenciam na adesão à propostas terapêuticas e no controle adequado de tratamento. Nesse contexto, este estudo torna-se relevante a medida que a verificação do diagnóstico de enfermagem selecionado proporciona o aprofundamento do conhecimento sobre esses fatores, e potencializa o processo de raciocínio diagnóstico com base em evidências clínicas. Dessa forma, o objetivo deste estudo foi identificar o diagnóstico de enfermagem Autocontrole Ineficaz da Saúde em pacientes dependentes do álcool.

\section{MATERIAL E MÉTODOS}

Trata-se de um estudo descritivo, do tipo transversal, com abordagem quantitativa, realizado em uma instituição hospitalar, localizada no interior do Estado de Pernambuco. O hospital desenvolve um programa de ações terapêuticas para pessoas com a Síndrome de Dependência do Álcool que permanecem na instituição por no máximo 90 dias. A média de tempo de internação é de 30 dias.

A amostra do estudo foi calculada a partir de métodos estatísticos, de acordo com os seguintes parâmetros: total de pacientes admitidos na unidade de internação com o diagnóstico médico de Síndrome da Dependência Alcoólica, no período de outubro de 2009 a março de 2010 ( $N=87)$; erro amostral de 6\%; coeficiente de confiança de $95 \%(Z a=1,96)$; e prevalência de $9 \%{ }^{(12)}$ para a dependência do álcool na região nordeste.
O tamanho amostral estimado foi de 46 indivíduos selecionados de forma consecutiva, à medida que preenchiam os seguintes critérios de inclusão: pacientes com idade acima de 18 anos e com o diagnóstico de Síndrome de Dependência do álcool. O único critério de exclusão foi apresentar-se em crise de abstinência.

A coleta de dados foi realizada por meio de entrevista estruturada, em espaço privativo, nos meses de abril e maio de 2010, por dois pesquisadores que utilizaram um instrumento composto por dados socioeconômicos e aspectos citados na literatura que apresentaram relação com as características definidoras e fatores relacionados do diagnóstico de enfermagem(13-15). Foi realizado um estudo piloto com cinco participantes e realizados os ajustes necessários no instrumento. Salienta-se que esses usuários não compuseram a amostra final do estudo.

As informações foram agrupadas com base nas características definidoras e fatores relacionados para se verificar a presença ou ausência do Autocontrole Ineficaz da Saúde. O processo de inferência diagnóstica foi realizado de acordo com a literatura e experiência clínica de três pesquisadores. A presença do diagnóstico foi determinada pelo conjunto das seguintes características: Falha em agir para reduzir fatores de risco, Escolhas na vida diária ineficazes para atingir as metas de saúde e Falha em incluir regimes de tratamento à vida diária.

Os dados foram analisados com o apoio do pacote estatístico SPSS (Statistical Package for the Social Sciences) versão 17.0 e estão apresentados em tabelas com as respectivas estatísticas descritivas e valores dos testes utilizados considerando o nível de significância de $5 \%$.

O estudo foi submetido ao Comitê de Ética da Universidade Federal de Pernambuco (CEP/CCS/UFPE) e obteve parecer favorável por meio do número do protocolo $047 / 10$. Foram atendidos os aspectos contidos na resolução 196/96 sobre Pesquisa com Seres Humanos do Conselho Nacional de Saúde/Ministério da Saúde.

\section{RESULTADOS}

Quanto ao perfil dos participantes, observou-se que todos são do sexo masculino e o predomínio de pessoas solteiras (39,1\%). A média de idade foi de 44,28 anos $( \pm 11,11)$. Com relação à escolaridade, $54,3 \%$ apresentaram ensino fundamental incompleto. Referente à ocupação, 34,8\% são autônomos, 37\% 
referiram exercer atividade formal e afirmaram que o uso do álcool interfere no desempenho das atribuições. $O$ desemprego foi referido por aproximadamente $24,0 \%$ dos participantes e o consumo descontrolado da substância foi citado como a causa da demissão. Quanto à realização de tratamentos anteriores, 39,1\% não buscou nenhuma forma de atendimento. E entre aqueles que procuraram, as alternativas de tratamento mais realizadas foram desintoxicação $(45,7 \%)$ e grupo de autoajuda $(45,7 \%)$.

De acordo com os dados da Tabela 1, a característica definidora mais frequente foi Expressão de desejo de controlar a doença (73,9\%). Dentre os indicadores utilizados para a sua mensuração, os que apresentaram maiores percentuais foram participação em atividades terapêuticas $(58,7 \%)$ e verbalização de interesse em dar continuidade ao tratamento (50,0\%).

Tabela 1: Distribuição dos indicadores das características definidoras do diagnóstico de enfermagem Autocontrole Ineficaz da Saúde em pacientes dependentes do álcool. Vitória de Santo Antão, PE, Brasil, 2010.

\begin{tabular}{lcc}
\hline Indicadores das características definidoras & $\mathrm{N}$ & $\%$ \\
\hline Expressão de desejo de controlar a doença & $\mathbf{3 4}$ & $\mathbf{7 3 , 9}$ \\
Participação de atividades terapêuticas & 27 & 58,7 \\
Verbaliza interesse em dar continuidade ao tratamento & 23 & 50,0 \\
Relato de desejo de cessar o consumo de álcool & 20 & 43,5 \\
Procurou tratamento voluntariamente & 19 & 41,3 \\
Utiliza estratégias que não envolvem o uso de álcool para lidar com os sentimentos negativos & 7 & 15,2 \\
\hline Escolhas na vida diária ineficazes para atingir as metas de saúde & $\mathbf{2 7}$ & $\mathbf{5 8 , 7}$ \\
Uso da bebida alcoólica para minimizar sentimentos negativos & 41 & 89,1 \\
Comparece continuamente a lugares propícios para o uso de álcool & 28 & 60,9 \\
Internação por coerção familiar & 5 \\
Não deseja participar das atividades terapêuticas & 10,9 \\
Falta de vontade de cessar o consumo de álcool & 2 \\
Desinteresse em dar continuidade ao tratamento & 4,3 \\
\hline Falha em agir para reduzir fatores de risco & 2 \\
Dificuldades de dialogar com o(a) parceiro(a) sobre o problema com o álcool & 4,3 \\
Dificuldades para manter relacionamento estável & 1 \\
Uso da bebida alcoólica (Razões para consumo - dependência) & $\mathbf{2 , 2}$ \\
Falta de estratégias para lidar com os sentimentos negativos & $\mathbf{1 6}$ \\
\hline Falha em incluir regimes de tratamento à vida diária & 23 & 50,0 \\
Recaídas (até duas) & 18 & 39,1 \\
Mais de duas tentativas de realização do tratamento & 11 & 23,9 \\
Mais de cinco tentativas de realização do tratamento & 5 \\
\hline Expressão de dificuldade com os regimes prescritos & 10,9 \\
Verbaliza complexidade com relação ao tratamento & $\mathbf{3 2 , 6}$ \\
\hline
\end{tabular}

Com relação às Escolhas na vida diária para atingir metas de saúde, observou-se a presença dessa característica em $58,7 \%$ dos alcoolistas e os aspectos que mostraram maior proporção para determinar a sua ocorrência foram utilizar a bebida alcoólica para minimizar sentimentos negativos $(89,1 \%)$ e frequentar continuamente lugares propícios para o uso dessa substância (60,9\%).

Falha em agir para reduzir fator de risco foi evidenciada em $34,8 \%$ dos participantes. Dificuldades para dialogar com o(a) parceiro(a) $(34,8 \%)$ e manter um relacionamento estável $(39,1 \%)$ apresentaram os maiores percentuais para inferir a presença desta característica.

Falha em incluir regimes de tratamento à vida diária foi mensurada a partir do relato de recaídas $(19,6 \%)$ e de outras tentativas de realização do tratamento $(26,1 \%)$. Assim, o fracasso para inserir o tratamento na rotina diária esteve presente em 32,6\% dos pacientes. Um percentual de $4,4 \%$ citou a complexidade do tratamento como atributo da Dificuldade com os regimes prescritos.

Quanto aos fatores relacionados descritos na Tabela 2 , os mais frequentes entre os 13 pacientes com o diagnóstico foram Suscetibilidade percebida (100,0\%), Conflito familiar (92,3\%) e Benefícios percebidos $(46,1 \%)$. 
Tabela 2: Distribuição dos indicadores dos fatores relacionados do diagnóstico de enfermagem Autocontrole Ineficaz da Saúde em pacientes dependentes do álcool. Vitória de Santo Antão, PE, Brasil, 2010.

\begin{tabular}{|c|c|c|}
\hline Fatores relacionados do diagnóstico de Enfermagem Autocontrole Ineficaz da Saúde & $\mathbf{N}$ & $\%$ \\
\hline Suscetibilidade percebida & 13 & 100,0 \\
\hline Conflito familiar & 12 & 92,3 \\
\hline Benefícios percebidos & 6 & 46,1 \\
\hline Déficit de apoio social & 5 & 38,5 \\
\hline Complexidade do sistema de assistência & 4 & 30,8 \\
\hline Déficit de conhecimento & 4 & 30,8 \\
\hline Dificuldades econômicas & 2 & 15,4 \\
\hline Complexidade do regime de tratamento & 1 & 7,7 \\
\hline Total de pacientes com o diagnóstico & \multicolumn{2}{|c|}{13} \\
\hline
\end{tabular}

Observou-se que ansiedade, presença rotineira em lugares propícios ao consumo, baixa auto-estima e isolamento social foram indicadores relevantes para a identificação do fator suscetibilidade percebida.

Referente ao conflito familiar, $58,7 \%$ relatou discussões e/ou brigas decorrentes do uso abusivo do álcool e dificuldades para dialogar sobre esse padrão de consumo. Em contrapartida, 50,0\% dos participantes reconhece a importância do tratamento e participam mais ativamente por acreditar em seus benefícios.

O déficit de suporte social, especialmente com relação à participação da família, foi citado por um paciente. Esse dado é importante, pois evidencia que, de modo geral, os familiares têm se inserido progressivamente no processo de reabilitação do alcoolista.

A análise da associação entre as características definidoras e o Autocontrole Ineficaz da Saúde demonstrou que Escolhas na vida diária ineficazes para atingir as metas de saúde, Expressão de desejo de controlar a doença e Falha em agir para reduzir fatores de risco estão relacionadas de forma significativa com o diagnóstico em questão (Tabela 3).

Tabela 3: Relação entre o diagnóstico de Enfermagem Autocontrole Ineficaz da Saúde e suas características definidoras em usuários dependentes do álcool. Vitória de Santo Antão, PE, Brasil, 2010.

\begin{tabular}{|c|c|c|c|c|}
\hline \multirow{2}{*}{\multicolumn{2}{|c|}{ Características Definidoras }} & \multicolumn{2}{|c|}{ Autocontrole Ineficaz da Saúde } & \multirow{2}{*}{ Valor p } \\
\hline & & Presente & Ausente & \\
\hline \multirow{2}{*}{$\begin{array}{l}\text { Escolhas na vida diária ineficazes para atingir as } \\
\text { metas de saúde }\end{array}$} & Presente & 8 & 8 & \multirow{2}{*}{$0,036^{*}$} \\
\hline & Ausente & 5 & 25 & \\
\hline \multirow{2}{*}{ Expressão de desejo de controlar a doença } & Presente & 12 & 15 & \multirow{2}{*}{$0,004 \dagger$} \\
\hline & Ausente & 1 & 18 & \\
\hline \multirow{2}{*}{ Falha em incluir regimes de tratamento à vida diária } & Presente & 10 & 3 & \multirow{2}{*}{$<0,001 *$} \\
\hline & Ausente & 3 & 28 & \\
\hline \multirow{2}{*}{ Falha em agir рага reduzir fatores de risco } & Presente & 3 & 12 & \multirow{2}{*}{$0,497 *$} \\
\hline & Ausente & 10 & 21 & \\
\hline \multirow{2}{*}{ Expressão de dificuldade com os regimes prescritos } & Presente & 1 & 1 & \multirow{2}{*}{$0,490 *$} \\
\hline & Ausente & 12 & 32 & \\
\hline Total de pacientes com o diagnóstico & & & 13 & \\
\hline
\end{tabular}

*Teste exato de Fisher; †Teste de Qui-quadrado.

\section{DISCUSSÃO}

Estudos associam a relação conjugal estável como fator que favorece a permanência do paciente no tratamento ${ }^{(14-16)}$. No entanto, nesta pesquisa observou-se o predomínio de pacientes solteiros.

A maioria dos entrevistados é autônoma e muitos estavam desempregados no momento da realização do estudo. Ser autônomo e/ou estar desempregado foi colocado como circunstância que proporciona uma maior dificuldade para manter-se vinculado, por longo prazo, às rotinas de tratamento. Esse fato associa-se negativamente à adesão ao regime terapêutico, uma vez que a autonomia confere um contexto de instabilidade para muitos e o desemprego figura como um atenuador ou muitas vezes como desencadeador de recaídas(15).

Pesquisas demonstraram que as dificuldades para aderir ao tratamento, quando se trata de pessoas dependentes de álcool, estão associadas com fatores que envolvem as áreas social, familiar e econômica ${ }^{(13-17)}$. Esses obstáculos foram evidenciados nas características 
definidoras e fatores relacionados identificados nesta amostra.

Quanto à característica Falha em agir para reduzir fatores de risco, sentimentos como ansiedade e baixa auto-estima frequente estão comumente associados com o Alcoolismo, fragilizam o dependente e contribuem substancialmente para a ocorrência de recaídas ${ }^{(16,18)}$. Outros achados sobre adesão ao tratamento do alcoolismo, mostraram que sintomas depressivos foram preditores da interrupção precoce do tratamento ${ }^{(19)}$. Além disso, o transtorno mental Depressão é reconhecido na literatura como um quadro que interfere na continuidade do tratamento(20).

A maioria dos entrevistados considerou o Alcoolismo uma doença, contudo acima de 50,0\% têm a concepção de que esse quadro clínico está relacionado com desvios de conduta e incapacidade do alcoolista de pensar por si próprio e tomar suas decisões de forma consciente. Isso sugere uma fragilidade de conhecimento dos participantes, fato já evidenciado em outro estudo(13). Além disso, o termo "fraqueza pessoal" foi encontrado nas falas dos usuários e remete a influência do pensar, ainda atual, da nossa sociedade que atribui ao alcoolista a culpa pela sua doença ${ }^{(21-22)}$.

Com relação às Escolhas na vida diária ineficazes para atingir as metas de saúde, viu-se que as principais razões para o consumo do álcool foram esquecer preocupações, eliminar a ansiedade e em virtude dos sintomas físicos da dependência. Pesquisas sobre a temática obtiveram achados divergentes, pois as primeiras razões encontradas estiveram relacionadas com os estados emocionais positivos (alegrar-se, relaxar) e posteriormente os negativos (diminuir ansiedade, esquecer preocupações)(19,23).

Quanto à busca por tratamento por coerção da família, observa-se, nessas circunstâncias, que o sucesso ou progresso referente ao regime terapêutico encontrase prejudicado. Pesquisadores mostraram que entre os fatores que se associam negativamente a adesão estão: falta de desejo de realizar o tratamento, redução do consumo por influência da família, internação por irritabilidade (crise de abstinência), agendamento de consultas realizado por terceiros e ausência nas consultas dentro de um programa de tratamento de dependentes de álcool(13).
Além disso, a falta de iniciativa é uma limitação que pode impedir a continuidade do tratamento, mas a resolutividade dependerá do nível de reflexão do paciente quanto ao seu problema e a vontade de enfrentá-lo(17).

Convém destacar o papel da família e dos amigos como possíveis agentes de detecção do uso abusivo de álcool e drogas, pois, na maioria dos casos, são esses entes próximos os que procuram ajuda para o dependente ${ }^{(24)}$. Ressalta-se que o envolvimento da família na reabilitação do alcoolista é importante e necessária, uma vez que são eles, em sua maioria, os responsáveis por cuidados em tempo integral(20).

Falha em incluir regimes de tratamento à vida diária traduz as dificuldades do tratamento e sugere também os prováveis motivos que levam ao consumo. O julgamento do paciente alcoolista de ter domínio sobre a ingestão do álcool, a necessidade de consumir a substâncias, a influência prejudicial dos amigos bebedores, a baixa autoestima, dentre outros, são fatores que contribuem para a recaída e demonstram a falsa crença de beber com controle ${ }^{(16)}$.

A falta de acompanhamento extra-hospitalar é um indicador importante para se analisar essa característica definidora e o fator relacionado complexidade do sistema de assistência. Observa-se que a organização estrutural da rede de apoio psicossocial aos pacientes em situação de sofrimento psíquico, tais como os dependentes do álcool e outras drogas, principalmente com relação ao acompanhamento após o processo de internação apresenta fragilidades.

Um estudo evidenciou a importância da articulação entre os equipamentos da atenção primária, os de saúde mental e a atenção específica a usuários de álcool e outras drogas e classifica esses dispositivos como potenciais e indispensáveis para a superação da lógica do modelo da psiquiatria clássica ${ }^{(24)}$.

Referente aos fatores relacionados, o mais frequente foi a suscetibilidade percebida e isso ocorre, provavelmente, devido à diversidade de fatores de risco que envolve o Alcoolismo. Um estudo já citado(16) encontrou uma média de 21 fatores que influenciam na recaída, entre eles estão ansiedade constante, frequentar lugares propícios ao consumo do álcool, influência de amigos e baixa auto-estima. 
Quanto ao conflito familiar, os participantes desta pesquisa relataram que se tornavam violentos quando alcoolizados e referiram dificuldades para dialogar com o(a) parceiro(a) sobre o seu padrão de uso da bebida alcoólica. Um estudo de revisão acerca do diagnóstico de enfermagem e processos familiares disfuncionais em alcoolistas, evidenciou que a desestrutura familiar e a violência doméstica estão entre as maiores dificuldades para lidar com o alcoolista ${ }^{(20)}$.

\section{CONSIDERAÇÕES FINAIS}

O diagnóstico de enfermagem Autocontrole Ineficaz da Saúde foi evidenciado em 13 pacientes e apresentou associação estatisticamente significante com as características definidoras Escolhas na vida diária ineficazes para atingir as metas de saúde, Falha em agir para reduzir fatores de risco e Expressão de desejo de controlar a doença. Os fatores relacionados mais

\section{REFERÊNCIAS}

1. World Health Organization. Global status report on alcohol and health [Internet]. Genebra: WHO; 2011 [acesso em: $20 \mathrm{dez}$ 2013]. Disponível em:

http://www.who.int/substance_abuse/publications/global_alco hol report/msbgsruprofiles.pdf.

2. INPAD - Instituto Nacional de Ciência e Tecnologia para Políticas do Álcool e Outras Drogas. II LENAD - Levantamento Nacional de Álcool e Drogas. Consumo de alcool no Brasil: tendências entre 2006/2012 [Internet]. São Paulo: INPAD; 2013 [acesso em: 20 dez 2013]. Disponível em:

http://inpad.org.br/wpcontent/uploads/2013/04/LENAD ALCOOL_ResultadosPreliminares.pdf.

3. Organização Mundial de Saúde: Classificação Estatística Internacional de Doenças e Problemas Relacionados à Saúde CID-10 [Internet]. Genebra: Organização Mundial de Saúde; 2008 [acesso em: 20 dez 2013]. Disponível em:

http://www.datasus.gov.br/cid10/V2008/cid10.htm.

4. Alves VS, Lima IMSO. Atenção à saúde de usuários de álcool e outras drogas no Brasil: convergência entre a saúde pública e os direitos humanos. Revista de Direito Sanitário [Internet]. 2013 [acesso em: $20 \mathrm{dez}$ 2013];13(3):9-32. Disponível em: http://www.revistas.usp.br/rdisan/article/view/56241. 5. Portaria GM/MS n 3088, de 23 de dezembro de 2011 (BR). Institui a Rede de Atenção Psicossocial para pessoas com sofrimento ou transtorno mental e com necessidades decorrentes do uso de crack, álcool e outras drogas, no âmbito do Sistema Único de Saúde (SUS). Diário Oficial da União [Internet]. $26 \mathrm{dez} 2011$ [acesso em: $20 \mathrm{dez}$ 2013]. Disponível em: http://bvsms.saude.gov.br/bvs/saudelegis/gm/2011/prt3088_2 3_12_2011 rep.html. 6. O'Farrell TJ, Clements K. Review of outcome research on marital and family therapy in treatment for alcoholism. J Marital Fam Ther [Internet]. 2012 [acesso em: $20 \mathrm{dez}$ 2013];38(1):12244. Disponível em: http://dx.doi.org/10.1111/j.17520606.2011.00242.x. frequentes foram Suscetibilidade percebida e Conflito familiar.

A análise desses aspectos fornece ao enfermeiro a possibilidade de identificar com maior precisão o fenômeno em estudo e propor intervenções mais efetivas, contribuindo para uma assistência ao alcoolista permeada pela ruptura de estigmas, fortalecimento de vínculos e respeito às singularidades.

Recomenda-se a realização de pesquisas longitudinais, com amostras maiores, que possam estabelecer relações mais acuradas entre as características definidoras e o diagnóstico de enfermagem. Ademais, sugere-se a investigação do diagnóstico citado em outros cenários de prática do enfermeiro, como por exemplo os CAPS - Ad, com o intuito de descrever como esse fenômeno se comporta quando se trata da reabilitação psicossocial do usuário de álcool e outras drogas.

7. Peixoto C, Prado CHO, Rodrigues CP, Cheda JND, Mota LBT, Veras AB. Impacto do perfil clínico e sociodemográfico na adesão ao tratamento de pacientes de um Centro de Atenção Psicossocial a Usuários de Álcool e Drogas (CAPSad). J Bras Psiquiatr [Internet]. 2010 [acesso em: 20 dez 2013];59(4):31721. Disponível em:

http://www.scielo.br/scielo.php?script=sci arttext\&pid=S004720852010000400008.

8. Alfaro-LeFevre RA. Aplicação do Processo de Enfermagem Uma ferramenta para o pensamento crítico. $7^{\text {a }}$ ed. Porto Alegre: Artmed; 2010.

9. NANDA Internacional. Diagnósticos de enfermagem da NANDA: definições e classificação 2012-2014. Porto Alegre: Artmed; 2012.

10. Freitas RWJF, Araújo MFM, Marinho NBP, Damasceno MMC, Caetano JA, Galvão MTG. Fatores relacionados ao diagnóstico de enfermagem autocontrole ineficaz da saúde entre diabéticos. Acta paul. enferm. [Internet] 2011 [acesso em: 20 dez 2013];24(3):365-72. Disponível em:

http://dx.doi.org/10.1590/S0103-21002011000300010.

11. Mendes LC, Sousa VEC, Lopes MVO. Acurácia das características definidoras do diagnóstico controle familiar ineficaz do regime terapêutico. Acta paul. enferm. [Internet] 2011 [acesso em: 20 dez 2013];24(2):219-24. Disponível em: http://dx.doi.org/10.1590/S0103-21002011000200010.

12. Secretaria Nacional de Políticas sobre Drogas, Presidência da República. I Levantamento Nacional sobre o Uso de Álcool, Tabaco e Outras Drogas entre Universitários das 27 Capitais Brasileiras [Internet]. Brasília: SENAD; 2010 [acesso em: $20 \mathrm{dez}$ 2013]. Disponivel em:

http://www.obid.senad.gov.br/portais/OBID/biblioteca/docume ntos/Publicacoes/Universitarios 2010/328160.pdf.

13. Fonsi Elbreder M, Carvalho De Humerez D, Laranjeira R.

Analysis of the obstacles related to treatment entry, adherence and drop-out among alcoholic patients. Riv Psichiatr [Internet] 2009 [acesso em: 20 dez 2013];44(6):351-6. Disponível em: http://dx.doi.org/10.1708/468.5535. 
14. Ribeiro MS, Ribeiro LC, Ferreira RA, Souza GF. Características de alcoólicos à adesão prolongada num programa ambulatório. Acta Med Port [Internet]. 2010 [acesso em: $20 \mathrm{dez}$ 2013];23(6):965-72. Disponível em: http://www.actamedicaportuguesa.com/revista/index.php/amp Larticle/view/753.

15. Ribeiro MS, Ribeiro LC, Garcia MA, Souza GF, Sousa KDC, Nogueira RB. Fatores associados a adesão a um programa de tratamento de alcoolistas. J Bras Psiquiatr [Internet]. 2008 [acesso em: 20 dez 2013];57(3):203-11. Disponível em: http://dx.doi.org/10.1590/S0047-20852008000300008. 16. Álvarez AMA. Fatores de risco que favorecem a recaída no alcoolismo. J Bras Psiquiatr [Internet]. 2007 [acesso em: $20 \mathrm{dez}$ 2013];56(3):188-93. Disponível em: http://dx.doi.org/10.1590/S0047-20852007000300006. 17. Monteiro CFS, Fé LCM, Moreira MAC, Albuquerque IEM, Silva MG, Passamani MC. Perfil sociodemográfico e adesão ao tratamento de dependentes de álcool em CAPS-ad do Piauí. Esc. Anna Nery [Internet]. 2011 [acesso em: $20 \mathrm{dez}$ 2013];15(1):90-5. Disponivel em: http://dx.doi.org/10.1590/S141481452011000100013.

18. Filizola CLA, Tagliaferro P, Andrade AS, Pavarini SCI, Ferreira NMLA. Alcoolismo e família: a vivência de mulheres participantes do grupo de autoajuda Al-Anon. J Bras Psiquiatr [Internet]. 2007 [acesso em: $20 \mathrm{dez} 2013$ ];58(3):181-6. Disponível em: http://dx.doi.org/10.1590/S004720852009000300007. 19. Corrêa Filho JM, Baltieri DA. Psychosocial and clinical predictors of retention in outpatient alcoholism treatment. Rev Bras Psiquiatr [Internet]. 2012 [acesso em: $20 \mathrm{dez}$ 2013];34(4):413-21. Disponível em: http://dx.doi.org/10.1016/j.rbp.2012.03.003. 20. Mangueira SO, Fernandes AFC, Pinheiro AKB, Oliveira Lopes MVO. Indicadores clínicos do diagnóstico de enfermagem processos familiares disfuncionais em alcoolistas: revisão integrativa. Rev. Eletr. Enf. [Internet]. 2013 [acesso em: $20 \mathrm{dez}$ 2013];15(3):819-28. Disponível em: http://dx.doi.org/10.5216/ree.v15i3.17766.

21. Vargas D, Luis MAV. Alcohol, alcoholism and alcohol addicts: conceptions and attitudes of nurses from district basic health centers. Rev Lat Am Enfermagem [Internet]. 2008 [acesso em: 20 dez 2013];16(esp):543-50. Disponível em: http://dx.doi.org/10.1590/S0104-11692008000700007. 22. Chagas M, Hildebrandt LM, Leite MT, Stumm EMF, Vianna RM. O alcoolismo e o grupo de Alcoólicos Anônimos: $O$ conhecimento de alcoolistas. Cadernos Brasileiros de Saúde Mental [Internet]. 2010 [acesso em: 20 dez 2013];2(4-5):190212. Disponivel em: http://www.incubadora.ufsc.br/index.php/cbsm/article/view/11 15. 23. Jora NP, Magalhães TR, Domingos JBC, Pillon SC. Campanha saúde na estrada: avaliação do padrão de consumo de álcool e do estresse. Rev. Eletr. Enf. [Internet]. 2010 [acesso em: $20 \mathrm{dez}$ 2013];12(1):37-46. Disponível em: http://dx.doi.org/10.5216/ree.v12i1.5526.

24. Souza LM, Pinto MG. Atuação do enfermeiro a usuários de álcool e de outras drogas na Saúde da Família. Rev. Eletr. Enf. [Internet]. 2012 [acesso em: 20 dez 2013];14(2):374-83.

Disponível em: http://dx.doi.org/10.5216/ree.v14i2.11245.

Artigo recebido em 28/08/2012.

Aprovado para publicação em 18/10/2013.

Artigo publicado em 31/12/2013. 